\title{
Glyoxalase-1 overexpression reduces endothelial dysfunction and attenuates early renal impairment in a rat model of diabetes
}

\author{
Olaf Brouwers • Petra M. G. Niessen • Toshio Miyata • Jakob A. Østergaard • \\ Allan Flyvbjerg • Carine J. Peutz-Kootstra • Jonas Sieber • Peter H. Mundel • \\ Michael Brownlee • Ben J. A. Janssen • Jo G. R. De Mey • Coen D. A. Stehouwer • \\ Casper G. Schalkwijk
}

Received: 26 April 2013 / Accepted: 2 October 2013 / Published online: 26 October 2013

(C) Springer-Verlag Berlin Heidelberg 2013

\begin{abstract}
Aims/hypothesis In diabetes, advanced glycation end-products (AGEs) and the AGE precursor methylglyoxal (MGO) are associated with endothelial dysfunction and the development of microvascular complications. In this study we used a rat model of diabetes, in which rats transgenically overexpressed the MGO-detoxifying enzyme glyoxalase-I (GLO-I), to determine the impact of intracellular glycation on vascular function and the development of early renal changes in diabetes. Methods Wild-type and Glol-overexpressing rats were rendered diabetic for a period of 24 weeks by intravenous
\end{abstract}

Electronic supplementary material The online version of this article (doi:10.1007/s00125-013-3088-5) contains peer-reviewed but unedited supplementary material, which is available to authorised users.

O. Brouwers $(\bowtie) \cdot$ P. M. G. Niessen • C. D. A. Stehouwer •

C. G. Schalkwijk

Laboratory for Metabolism and Vascular Medicine, Division of

General Internal Medicine, Department of Internal Medicine,

Maastricht University Medical Center, Universiteitssingel 50,

P.O. Box 616 (box 14), 6200 MD Maastricht, the Netherlands

e-mail: olaf.brouwers@maastrichtuniversity.nl

T. Miyata

Centre of Translational and Advanced Research, Tohoku University,

Sendai, Japan

J. A. Østergaard · A. Flyvbjerg

The Medical Research Laboratories, Department of Clinical

Medicine, Aarhus University, Aarhus, Denmark

\section{J. A. Østergaard · A. Flyvbjerg}

Department of Endocrinology and Internal Medicine,

Aarhus University Hospital, Aarhus, Denmark

J. A. Østergaard

The Danish Diabetes Academy, Aarhus, Denmark injection of streptozotocin. Mesenteric arteries were isolated to study ex vivo vascular reactivity with a wire myograph and kidneys were processed for histological examination. Glycation was determined by mass spectrometry and immunohistochemistry. Markers for inflammation, endothelium dysfunction and renal dysfunction were measured with ELISA-based techniques.

Results Diabetes-induced formation of AGEs in mesenteric arteries and endothelial dysfunction were reduced by Glo1 overexpression. Despite the absence of advanced nephrotic lesions, early markers of renal dysfunction (i.e. increased

\section{J. Peutz-Kootstra}

Department of Pathology, Maastricht University Medical Center, Maastricht, the Netherlands

J. Sieber · P. H. Mundel

Harvard Medical School and the Division of Nephrology,

Massachusetts General Hospital, Boston, MA, USA

M. Brownlee

International Center for Diabetic Complications Research, Albert Einstein College of Medicine, New York, NY, USA

B. J. A. Janssen · J. G. R. De Mey

Department of Pharmacology, Maastricht University Medical Center, Maastricht, the Netherlands

O. Brouwers • P. M. G. Niessen • B. J. A. Janssen • J. G. R. De Mey •

C. D. A. Stehouwer · C. G. Schalkwijk

Cardiovascular Research Institute Maastricht (CARIM),

Maastricht University, Maastricht, the Netherlands 
glomerular volume, decreased podocyte number and diabetesinduced elevation of urinary markers albumin, osteopontin, kidney-inflammation-molecule-1 and nephrin) were attenuated by Glol overexpression. In line with this, downregulation of Glol in cultured endothelial cells resulted in increased expression of inflammation and endothelium dysfunction markers. In fully differentiated cultured podocytes incubation with MGO resulted in apoptosis.

Conclusions/interpretation This study shows that effective regulation of the GLO-I enzyme is important in the prevention of vascular intracellular glycation, endothelial dysfunction and early renal impairment in experimental diabetes. Modulating the GLO-I pathway therefore may provide a novel approach to prevent vascular complications in diabetes.

Keywords Endothelial dysfunction - Glycation . Glyoxalase-1 · Methylglyoxal $\cdot$ Renal impairment

$\begin{array}{ll}\text { Abbreviations } & \\ \text { AGEs } & \text { Advanced glycation end-products } \\ \text { CEL } & N^{\varepsilon} \text {-(1-Carboxyethyl)lysine } \\ \text { CML } & N^{\varepsilon} \text {-(1-Carboxymethyl)lysine } \\ \text { 3-DG } & \text { 3-Deoxyglucosone } \\ \text { eNOS } & \text { Endothelial nitric oxide synthase } \\ \text { GO } & \text { Glyoxal } \\ \text { GLO-I } & \text { Glyoxalase-I } \\ \text { ICAM-1 } & \text { Intercellular adhesion molecule-1 } \\ \text { KIM-1 } & \text { Kidney injury marker-1 } \\ \text { MAP } & \text { Mean arterial pressure } \\ \text { MCP-1 } & \text { Monocyte chemotactic protein-1 } \\ \text { MG-H1 } & N^{\delta} \text {-(5-Hydro-5-methyl-4-imidazolon- } \\ & \text { 2-yl)-L-ornithine } \\ \text { MGO } & \text { Methylglyoxal } \\ \text { NO } & \text { Nitric oxide } \\ \text { NRF-2 } & \text { Nuclear factor (erythroid-derived 2)-like 2 } \\ \text { qPCR } & \text { Quantitative PCR } \\ \text { siRNA } & \text { Small-interfering RNA } \\ \text { SNP } & \text { Sodium nitroprusside } \\ \text { STZ } & \text { Streptozotocin } \\ \text { UPLC-MSMS } & \text { Ultra-performance liquid chromatography } \\ & \text { tandem mass spectrometry } \\ \text { VCAM-1 } & \text { Vascular cell-adhesion molecule-1 } \\ \text { WT-1 } & \text { Wilms' tumour-1 }\end{array}$

\section{Introduction}

Hyperglycaemia is a key risk factor for microvascular complications leading to excess morbidity and mortality in diabetic patients [1]. Microangiopathy, damage to the small blood vessels and capillaries, is a direct result of chronic hyperglycaemia and is the main cause of major complications such as retinopathy and nephropathy [2,3]. Dysfunction of the vascular endothelium is considered to be an important factor in the initiation, progression and clinical outcome of diabetic microangiopathy [4]. One hypothesis as to how hyperglycaemia leads to endothelial dysfunction and, consequently, vascular complications is the formation of advanced glycation end-products (AGEs) [5].

AGEs are a heterogeneous family of non-enzymatically modified proteins, which are increased in patients with diabetes. In addition to the formation of AGEs by carbohydrates in the classical Maillard reaction, intracellular glucose-derived glycolytic intermediates, such as methylglyoxal (MGO), glyoxal (GO) and 3-deoxyglucosone (3-DG), also form AGEs [6]. Because these intracellular dicarbonyl compounds form many more glycated proteins and at a faster rate than do equimolar amounts of glucose, intracellular AGE formation is thought to play an important role in the link between AGEs and diabetic complications. Indeed, intracellular AGEs have been implicated in activating intracellular signalling pathways as well as in modifying the function of intracellular proteins, thereby contributing to diabetic vascular complications [7]. The very reactive oxoaldehyde MGO has been identified as the major precursor in the formation of intracellular AGEs in endothelial cells [8]. MGO is formed mainly by fragmentation of the triose phosphates glyceraldehyde-3-phosphate and dihydroxyacetone-phosphate and is, under physiological circumstances, efficiently detoxified to D-lactate by the glyoxalase system, in which glyoxalase-I (GLO-I) is the rate limiting enzyme [9]. MGO is known to have detrimental effects on cellular function and it is also evident that elevated levels of MGO are responsible for renal oxidative stress, as demonstrated in diabetic rats [10]. Most importantly, progression of hypertension and diabetic nephropathy in humans is also significantly related to increased levels of MGO [11, 12].

We previously showed in a rat model of diabetes that Glo1 overexpression detoxifies $\mathrm{MGO}$ and $\mathrm{GO}$, and thereby decreases AGEs and markers of oxidative stress [13]. Furthermore, we recently demonstrated in an ex vivo model that high glucose levels and short-term diabetes-induced impairment of endothelium-dependent vasorelaxation in rat mesenteric arteries are mediated by intracellular MGO levels [14]. In addition, Glol overexpression protects against diabetes-induced retinal neuroglial and vasodegenerative pathology [15]. However, the involvement of the major AGE precursor MGO in long-term diabetes-induced endothelial dysfunction and, consequently, the development of renal microvascular complications remain largely unknown.

Therefore, the present study explored the effect of Glo1 overexpression on microvascular and renal function 24 weeks after streptozotocin (STZ)-induced diabetes in the rat. We hypothesised that elevated levels of MGO are involved in 
microvascular dysfunction and in the development of diabetic renal changes, and that Glo1 overexpression can prevent this.

\section{Methods}

Animal model Heterozygous transgenic Glol -overexpressing rats were crossed with wild-type Wistar rats (Nippon Seibutsu Zairyo Center, Saitama, Japan) to obtain enough Glo1overexpressing progeny for the experiment [13]. Young adult wild-type and Glo1 transgenic male rats (10 weeks of age) were rendered diabetic for a period of 24 weeks by a single intravenous injection of STZ (Sigma-Aldrich, Zwijndrecht, the Netherlands; $45 \mathrm{mg} / \mathrm{kg}$ body weight). Only rats with fasting blood glucose levels above $15 \mathrm{mmol} / \mathrm{l}$ were included in the study (Contour test strips, Bayer Health Care, Leverkusen, Germany). Weight- and age-matched control wild-type rats were not injected. The rats were divided into three groups: wild-type control group (WtC; $n=9$ ), wild-type STZ-induced diabetic group (WtD; $n=8)$ and Glo1overexpressing STZ-induced diabetic group (TgD; $n=13)$.

The study protocols were approved by the Committee on Animal Experimentation and Welfare of Maastricht University.

Twenty-four weeks after the induction of diabetes, rats were killed and mesenteric arteries, kidneys, urine and EDTA blood were processed for further analysis.

Blood pressure and heart rate measurement Under isoflurane (Baxter, Deerfield, IL, USA) anaesthesia a PE-10 catheter was inserted in the abdominal aorta via the femoral artery and connected to a pressure transducer (Miller Instruments, Houston, TX, USA). The pressure signal was digitally sampled at $2 \mathrm{kHz}$ and mean arterial pressure (MAP) and heart rate were calculated over a $10-15$ min time period after haemodynamic variables were stabilised.

Measurement of GLO-I activity GLO-I activity was assayed by spectrophotometry (Synergy, BioTek, Winooski, VT, USA), by monitoring the increase in absorbance at $240 \mathrm{~nm}$ due to the formation of $S$-D-lactoylglutathione for $10 \mathrm{~min}$ at $37^{\circ} \mathrm{C}$ according to the method of McLellan and Thornalley [16].

Measurement of vascular function, morphology and gene expression Vascular reactivity was measured as described earlier [14]. For further details see electronic supplementary materials [ESM] Methods.

On paraformaldehyde-fixed mesenteric arteries, media cross-sectional area, media thickness and lumen diameter were determined using Leica Qwin software (Leica, Groot Bijgaarden, Belgium) on $4 \mu \mathrm{m}$ sections stained with Lawson's solution (Boom, Meppel, the Netherlands), a classic elastin stain. To stain collagen, cross-sections were deparaffinised and incubated in phosphomolybdenic acid $(0.2 \% \mathrm{wt} / \mathrm{vol}$.) for $5 \mathrm{~min}$, followed by incubation with Sirius red (SigmaAldrich). After washing with $0.1 \mathrm{~mol} / \mathrm{l} \mathrm{HCl}$ for $2 \mathrm{~min}$, sections were dehydrated and protected with coverslips. Collagen content was calculated as the percentage collagen-positive area per total area.

After homogenisation of vascular tissue by the use of glass beads and a mini-bead beater, total cellular RNA was extracted using TRIzol isolation (Invitrogen, Life Technologies, Paisley, UK). Reverse transcription was performed with a Reverse Transcriptase kit from Invitrogen. Subsequently, quantitative PCR (qPCR) assays were performed using Quanta Universal PCR MasterMix on an ABI PRISM 7900 HT (Applied Biosystems, Foster City, CA, USA) and a primer of the target (i.e. vascular cell-adhesion molecule-1 [VCAM-1], intercellular adhesion molecule-1 [ICAM-1], endothelial nitric oxide synthase [eNOS]) or a primer of the housekeeping gene, $\beta$-actin, all purchased from Eurogentec (Liège, Belgium).

Immunohistochemical and histological examinations Mesenteric arteries and kidneys were fixed with phosphatebuffered ( $\mathrm{pH}$ 7.4) formaldehyde ( $4 \%$ vol./vol.) overnight at room temperature. Subsequently, arteries and kidneys were transferred to $70 \% \mathrm{vol} . / \mathrm{vol}$. ethanol, embedded in paraffin and processed for histological examination.

Anti-GLO-I $\left(1: 1,000\right.$; see ESM Methods), anti- $N^{\varepsilon}$ (1-carboxymethyl)lysine (CML) (1:4,000; homemade) [17], anti- $N^{\delta}$-(5-hydro-5-methyl-4-imidazolon-2-yl)-L-ornithine (MG-H1) (1:100,000, generous gift from M. Brownlee), antieNOS (1:5,000; BD transduction laboratories, Breda, the Netherlands), anti-Wilms' tumour-1 (WT-1) (undiluted; DAKO, Glostrup, Denmark), and anti-active-caspase-3 (1:200; Cell Signaling Technology, Danvers, MA, USA) were used as primary antibodies. After washing in PBS (pH 7.4), sections were incubated for $30 \mathrm{~min}$ with the appropriate biotin-labelled antibody (1:500) at room temperature and subsequently washed in PBS. After incubation with streptavidin-horseradish peroxidase (1:200, DAKO) for $60 \mathrm{~min}$ at room temperature, peroxidase was visualised with 3,3-diamino-benzidine-tetrahydrochloride $/ \mathrm{H}_{2} \mathrm{O}_{2}$ (SigmaAldrich) for 3-5 min. Immunostaining was scored for anatomical localisation and its intensity or number of positive cells. For the intensity scoring, each positive vessel was given a score: 0 , absent; 1 , weak positivity; 2 , moderate positivity; 3 , strong positivity. Each multiplication score was then added and the sum was divided by the number of samples, resulting in an immunohistochemical score. All stainings were scored by two blinded observers.

The middle part of the right kidney (containing the papilla) was embedded in paraffin for examination. Two micron-thick sections were cut on a rotation microtome and stained with 
$p$-aminosalicylic acid and haematoxylin (DAKO). Total glomerulus volume was measured observer-blinded at a magnification of $\times 400$ as previously described [18].

Creatinine measurement in plasma and urine Urinary and plasma creatinine levels were measured by ultraperformance liquid chromatography tandem mass spectrometry (UPLC-MSMS) (UPLC Acquity and Micromass Quattro Premier XE Tandem Mass Spectrometer; Waters, Milford, MA, USA) as described elsewhere [19]. Internal standard creatinine (methyl-d3) (CDN Isotopes, Quebec, Canada) was mixed with deproteinised plasma or diluted urine and subsequently analysed by UPLC-MSMS.

Biomarker detection Rat kidney injury panel 1 was purchased from Meso Scale discovery (Rockville, MD, USA). This assay detects lipocalin-2, osteopontin and kidney-injurymolecule (KIM-1) in a sandwich immunoassay and uses a competitive assay format to detect albumin. Each 96-well plate had four carbon electrodes in the bottom of each well; each pre-coated with one of the four anti-kidney-injury marker antibodies of interest. The intra-assay variations of albumin, lipocalin-2, osteopontin and KIM-1 were 5.0\%, 4.0\%, 8.4\% and $3.3 \%$, respectively. Monocyte chemotactic protein-1 (MCP-1) was measured with MSD kit K151AYB-2 (Meso Scale) according to the manufacturer's protocol (intra-assay variation was 3.3\%). The markers ICAM-1 (Diaclone, Ann Arbor, MI, USA), IL-6 (Sanquin, Amsterdam, the Netherlands) and nephrin (Exocell, Philadelphia, PA, USA) were measured using standard ELISA procedures (according to the manufacturer's protocol) and the intra-assay variations were $2.8 \%, 1.6 \%$ and $9.1 \%$ respectively.

Plasma nitrite and nitrate levels were determined using a commercially available colorimetric assay kit (Cayman
Chemical, Ann Arbor, MI, USA) based on the Griess reaction. Before the assay, the plasma samples were ultrafiltered though a $30 \mathrm{kDa}$ molecular mass cut-off filter (Ultrafree-MC centrifugal filter units; Millipore Corporation, Bedford, MA, USA) to reduce the background absorbance caused by haemoglobin.

Measurement of AGEs and oxo-aldehydes The AGEs CML, $N^{\varepsilon}$-(1-carboxyethyl)lysine (CEL) and MG-H1 were measured using UPLC-MSMS (Waters) as described earlier [20]. Oxoaldehydes GO, MGO and 3-DG in plasma were assayed by derivatisation with $o$-phenylenediamine and UPLC-MSMS [21].

In vitro cell culture experiments Human immortalised endothelial cells (ECRF-24) were cultured for Glo1 silencing experiments and conditionally immortalised mouse podocytes were cultured for apoptosis assays. For further details see ESM Methods.

Statistics All values are expressed as mean \pm SEM and $p<$ 0.05 was considered statistically significant. For further details see ESM Methods.

\section{Results}

General physical and biochemical characteristics Injection of the rats with STZ produced characteristic symptoms of diabetes, including hyperglycaemia, decreased body weight gain, hyperphagia, polydipsia and polyuria, without any differences between Glol genotypes (Table 1). Diabetes resulted in significant increased levels of GO, MGO and
Table 1 General physical and biochemical characteristics of the rats after 24 weeks of diabetes

${ }^{*} p<0.05$ compared with $\mathrm{WtC}$; $* * * p<0.001$ compared with $\mathrm{WtC} ;{ }^{\dagger} p<0.05$ compared with WtD

\begin{tabular}{|c|c|c|c|}
\hline Characteristic & $\mathrm{WtC}$ & $\mathrm{WtD}$ & $\mathrm{TgD}$ \\
\hline Plasma glucose $(\mathrm{mmol} / \mathrm{l})$ & $6 \pm 1$ & $32 \pm 1^{*}$ & $31 \pm 1 *$ \\
\hline Body weight (g) & $475 \pm 12$ & $300 \pm 11 *$ & $300 \pm 12 *$ \\
\hline Food intake (g) & $19 \pm 2$ & $43 \pm 2 *$ & $43 \pm 2 *$ \\
\hline Fluid intake (ml/day) & $19 \pm 2$ & $187 \pm 14^{*}$ & $200 \pm 13 *$ \\
\hline Urine production (ml/day) & $11 \pm 1$ & $183 \pm 15^{*}$ & $207 \pm 11^{*}$ \\
\hline $\mathrm{GO}(\mu \mathrm{mol} / \mathrm{l})$ & $0.40 \pm 0.03$ & $0.81 \pm 0.10^{*}$ & $0.66 \pm 0.02^{\dagger}$ \\
\hline $\mathrm{MGO}(\mu \mathrm{mol} / \mathrm{l})$ & $0.33 \pm 0.02$ & $0.64 \pm 0.07^{*}$ & $0.50 \pm 0.02^{\dagger}$ \\
\hline 3-DG $(\mu \mathrm{mol} / \mathrm{l})$ & $3.2 \pm 0.1$ & $6.1 \pm 0.2 *$ & $6.1 \pm 0.2 *$ \\
\hline Urinary D-lactate $(\mu \mathrm{mol} / \mathrm{mmol}$ creatinine $)$ & $1 \pm 0.3$ & $196 \pm 63^{*}$ & $568 \pm 237 * * *$ \\
\hline CML (pmol/mg) & $4.8 \pm 0.5$ & $10.8 \pm 1.1^{*}$ & $10.4 \pm 1.2^{*}$ \\
\hline CEL (pmol/mg) & $7.5 \pm 0.8$ & $15.0 \pm 1.4^{*}$ & $13.5 \pm 1.4^{*, \dagger}$ \\
\hline MG-H1 (pmol/mg) & $206 \pm 10$ & $244 \pm 11 *$ & $199 \pm 9^{\dagger}$ \\
\hline MAP (mmHg) & $91 \pm 2$ & $62 \pm 2 *$ & $76 \pm 3 *, \dagger$ \\
\hline Heart rate (bpm) & $340 \pm 12$ & $261 \pm 4^{*}$ & $284 \pm 9 *$ \\
\hline
\end{tabular}


3-DG. Glo1 overexpression decreased GO and MGO, but not 3-DG. The increased GLO-I activity in diabetes, and predominantly in the transgenic rats, was accompanied by significant increased levels of urinary D-lactate, the detoxified metabolite of MGO. In addition, 24 weeks of diabetes also significantly increased the levels of CML, CEL and MG-H1 in plasma. Both CEL and MG-H1 were significantly lower in the transgenic rats while CML was not altered (Table 1).

Both MAP and heart rate were decreased in the wild-type diabetic rats compared with the control rats (Table 1). The decrease in blood pressure after 24 weeks of diabetes was significantly attenuated by Glol overexpression, although heart rate was not statistically influenced by the overexpression of Glo1.

Diabetes-induced AGE formation in rat mesenteric arteries is prevented by Glo1 overexpression The mesenteric arteries of the Glol-overexpressing rats showed an increase in GLO-I protein levels and GLO-I activity after 24 weeks of diabetes as compared with control and wild-type diabetic rats (Fig. 1a-d). The immunohistochemical stainings for CML (Fig. 1e-h) and
MG-H1 (Fig. 1i-1) in rat mesenteric arteries were both significantly elevated in the wild-type diabetic group as compared with the control rats. Staining for MG-H1 was predominantly localised in the endothelial cells, while CML was also present in the smooth muscle cells and adventitia. Overexpression of Glo1 prevented this diabetes-induced elevation of both CML and MG-H1 in the diabetic mesenteric arteries.

Morphological analyses of the mesenteric arteries showed no differences in mesenteric lumen radius, media crosssectional area or media thickness after 24 weeks of diabetes (see ESM Table 1). Analyses of the mesenteric arteries for collagen deposition also showed no significant differences in collagen content corrected for medial area between the different groups.

Diabetes-induced impairment of vasorelaxation in rat mesenteric arteries is reduced by Glol overexpression To investigate the function of the resistance arteries in diabetes and the effect of Glol overexpression, we measured endothelium-dependent and -independent vasorelaxation in a

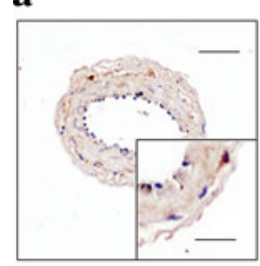

b

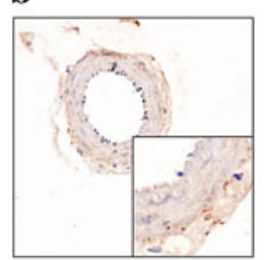

e

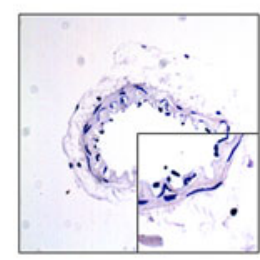

f

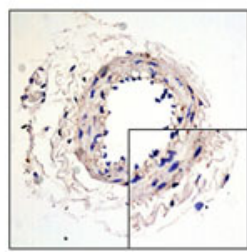

i

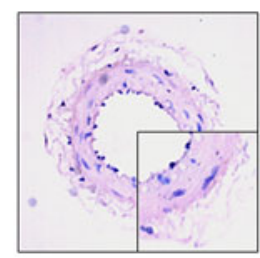

j

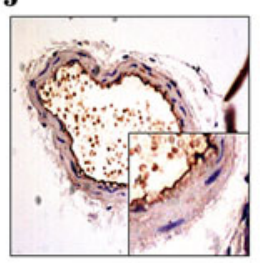

c

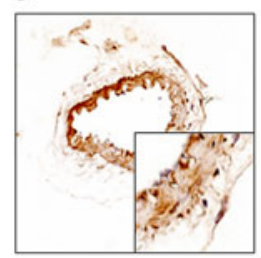

g

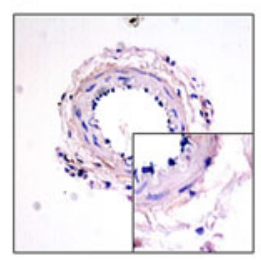

k

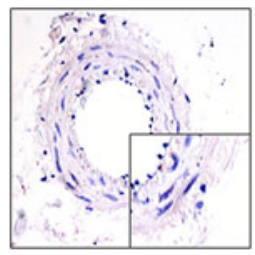

d

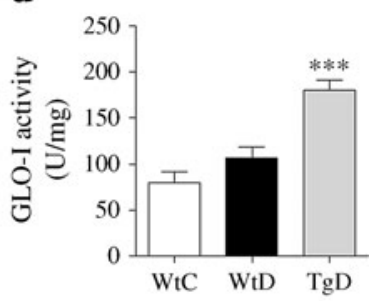

h

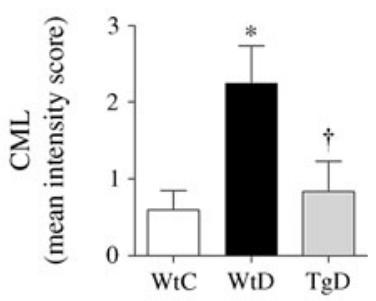

I

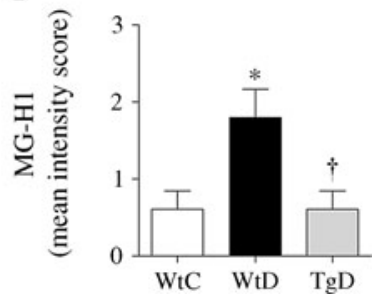

Fig. 1 Immunohistochemical staining of GLO-I (a-c) and the GLO-I enzyme activity (d) showed a functionally active overexpression of GLO-I in mesenteric arteries in GLO-I STZ rats (TgD) compared with wild-type control (WtC) and wild-type diabetic (WtD) rats $(* * * p<$ 0.001 ). Units $(\mathrm{U})$ in $\mathrm{nmol} / \mathrm{min}$. (e-h) Diabetes resulted in increased levels of CML staining (f) in isolated rat mesenteric arteries compared with control (e). Quantification of these stainings in arbitrary units (h) showed an increase in CML after 24 weeks of diabetes $\left({ }^{*} p<0.05\right.$, WtD vs $\left.\mathrm{WtC}\right)$, which could be normalised by Glol overexpression $\left({ }^{\dagger} p<0.05\right.$, TgD vs $\mathrm{WtD})(\mathbf{g})$. (i-l) Diabetes resulted in increased levels of MG-H1 staining in isolated rat mesenteric arteries (j) compared with control (i). Quantification of these stainings in arbitrary units (I) showed an increase in MG-H1 after 24 weeks of diabetes $\left({ }^{*} p<0.05\right.$, WtD vs WtC), which was normalised by GLO-I overexpression ( ${ }^{\dagger} p<0.05, \mathrm{TgD}$ vs WtD) (k). Scale bars in (a) $100 \mu \mathrm{m}$ in the main image and $50 \mu \mathrm{m}$ in the insert 
isolated second-order mesenteric arteries of the rats in a wire myograph. Maximum contraction did not differ between the groups after 24 weeks of diabetes (active wall tension for wild-type control, wild-type diabetic and Glo1overexpressing diabetic rats was $3.6 \pm 0.3,3.7 \pm 0.3$ and $3.3 \pm$ $0.3 \mathrm{~N} / \mathrm{m}$, respectively). Diabetes resulted in a decrease in total acetylcholine-induced, endothelium-dependent, nitric oxide (NO)-mediated and also NO-independent vasorelaxation after 24 weeks of diabetes (Fig. 2a-c). Sodium nitroprusside (SNP)-induced endothelium-independent vasorelaxation was not altered due to diabetes (Fig. 2d). Decreased endotheliumdependent reactivity of the mesenteric arteries of the diabetic rats was significantly improved by Glol overexpression (for potency and efficacy values see ESM Table 2).

Modulation of GLO-I activity regulates the expression of endothelial activation and inflammation markers We next investigated the effect of GLO-I modulation on other markers of endothelial dysfunction. We found increased expression of the endothelial dysfunction markers VCAM-1 and ICAM-1 in the diabetic rat mesenteric arteries $(p<0.05)$. The increases in the mRNA levels of these markers were completely prevented
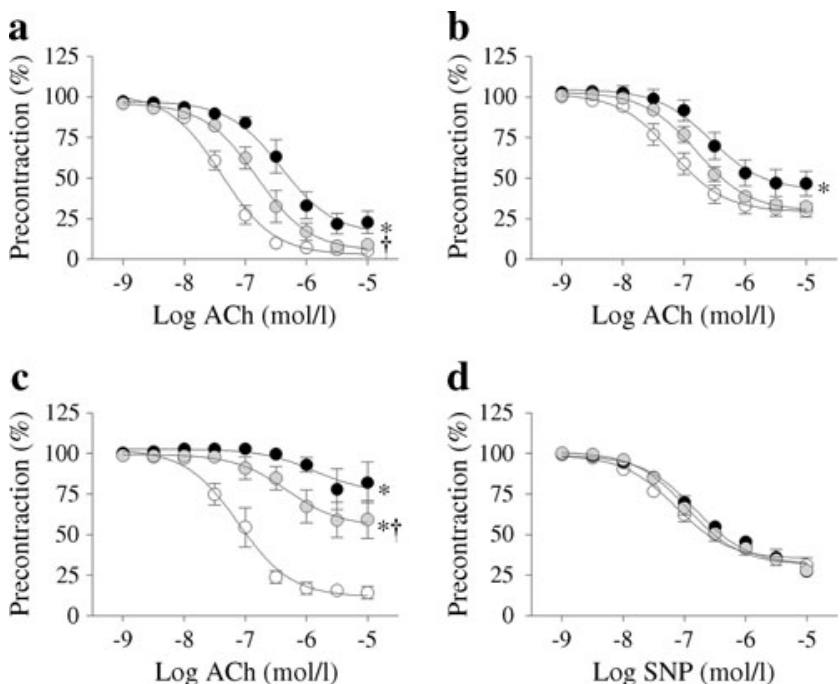

Fig. 2 White circles, control (WtC) rats; black circles, diabetic (WtD) rats; grey circles, GLO-I diabetic (TgD) rats; $\mathrm{ACh}$, acetylcholine. $\log _{10}$ transformed data are given on the x-axis. (a) Diabetes resulted in a statistically significant rightward shift of the dose-response curve to ACh $(* p<0.05$, compared with $\mathrm{WtC})$. Overexpression of Glo1 partially prevented this impairment $\left({ }^{\dagger} p<0.05\right.$, TgD vs WtD). (b) The concentration-response curve of the vasorelaxation due to endotheliumderived NO was significantly shifted after 24 weeks of diabetes $\left({ }^{*} p<\right.$ 0.05 , compared with $\mathrm{WtC}$ ). Overexpression of Glol resulted in a trend toward improvement, but was only borderline significant after 24 weeks of diabetes $(p=0.08)$. (c) The concentration-response curve of the vasorelaxation due to eNOS-independent relaxation was also significantly shifted after 24 weeks of diabetes $\left({ }^{*} p<0.05\right.$, compared with $\mathrm{WtC})$. Overexpression of Glol resulted in a significant improvement after 24 weeks of diabetes $\left({ }^{\dagger} p<0.05, \mathrm{TgD}\right.$ vs WtD). (d). Endotheliumindependent SNP-induced vasorelaxation was not altered after 24 weeks of diabetes by Glo1 overexpression (Fig. 3a, b). However, the increase in plasma ICAM-1 in the wild-type diabetic group $(22.9 \pm 1.4 \mathrm{vs}$ $13.9 \pm 0.6 \mathrm{ng} / \mathrm{ml}$ ), could not be corrected by Glol overexpression $(26.0 \pm 1.8 \mathrm{ng} / \mathrm{ml})$, indicating a local effect of GLO-I. Furthermore there were no significant effects of diabetes or Glo1 overexpression on Nos 3 mRNA expression (Fig. 3c) or protein levels (Fig. 3e-h) in the mesenteric arteries. Nevertheless total plasma levels of the NO metabolites nitrite and nitrate (NOx) were significantly decreased in the wild-type diabetic rats, an effect which was prevented in the Glol-overexpressing rats (Fig. 3d).

In line with the in vivo data, we observed that in cultured human endothelial cells downregulation of Glo1 resulted in the upregulation of several genes involved in pathways of vascular dysfunction and inflammation. Small-interfering RNA (siRNA) against Glol resulted in a decrease in Glol expression (Fig. 4a) and GLO-I activity (Fig. 4b) by more than $60 \%$. This decrease in GLO-I activity resulted in dysfunction of the cultured endothelial cells, as indicated by a mild but significant decrease in endothelial cell viability (Fig. 4c), and increased expression of genes representing endothelial inflammation (Fig. 4d) and activation (Fig. 4e). Protein levels of MCP-1, IL-6 and ICAM-1 were also elevated in the supernatant fractions of the cultured endothelial cells and supported the results of the qPCR (Fig. $4 \mathrm{f}-\mathrm{h}$ ).

Markers of early renal damage are attenuated by Glo1 overexpression after 24 weeks of diabetes Because endothelial dysfunction and inflammation are considered to be important factors in the initiation and progression of vascular complications, we next determined the effect of Glo1 overexpression on the development of diabetesinduced renal dysfunction. In our rat model, 24 weeks of diabetes resulted in nephromegaly, which tended to be decreased by Glo1 overexpression (Table 2). Detailed morphometric analyses of the wild-type diabetic kidneys showed a statistically significant increase in glomerular volume, which was not significant, of the diabetic kidneys from Glo1-overexpressing rats compared with the controls. Furthermore, the wild-type diabetic rats showed signs of renal hyperfiltration, as indicated by the increase in body-weightcorrected creatinine clearance.

Immunohistochemical analyses showed increased presence of CML and MG-H1 in the mesangial matrix and peritubular capillaries of the diabetic rats, which could not be prevented by Glol overexpression. This observation was also confirmed by UPLC-MSMS analysis of total kidney lysates (data not shown). Furthermore, extensive histological analysis of the kidney did not show any collagen deposition or fibrotic or inflammatory differences in glomerular, tubulointerstitial or capillary tissue between the three groups after 24 weeks of diabetes (ESM Fig. 1). 

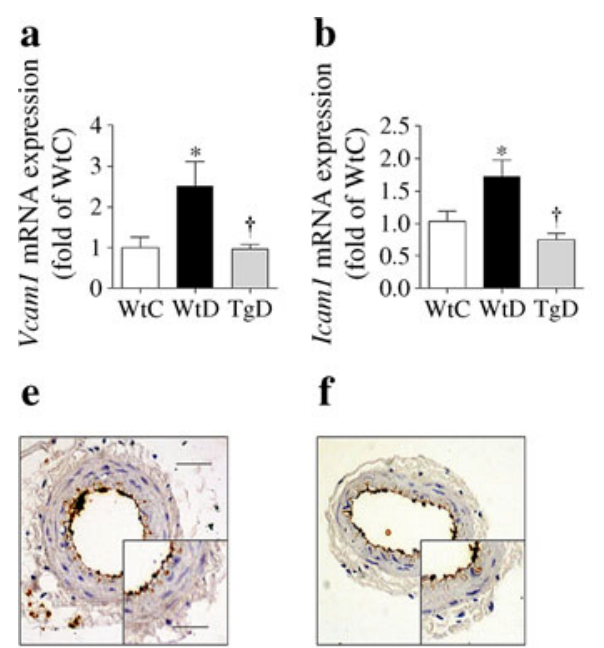

Fig. 3 Both Vcam1 (a) and Icam1 (b) mRNA expression levels in the mesenteric arteries of wild-type diabetic rats (WtD) were elevated after 24 weeks of diabetes $(* p<0.05$, compared with control [WtC]), and Glol overexpression (TgD) prevented this $\left({ }^{\dagger} p<0.05, \mathrm{TgD}\right.$ vs WtD). The mRNA expression levels of eNOS (Nos 3 mRNA) (c) were not altered by either diabetes or Glol overexpression; plasma levels of NO

We next focused on early markers of nephropathy in the diabetic rats. Immunohistological staining of renal podocytes showed a decrease in number in the wild-type diabetic glomeruli (Fig. 5b) compared with controls (Fig. 5a). Glo1 overexpression significantly attenuated this decrease (Fig. 5c, d). Increased staining in the diabetic glomeruli (Fig. 5e, f) of the apoptotic marker active-caspase-3 indicates that this decrease in podocyte number is, at least partially, apoptosis-dependent and that this could be prevented by Glol overexpression (Fig. $5 \mathrm{~g}, \mathrm{~h}$ ). Subsequently, Glo1 overexpression also partially prevented

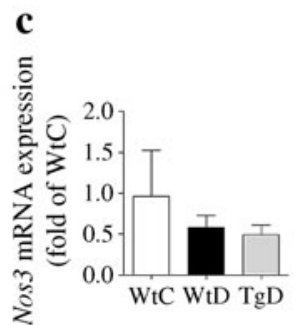

d
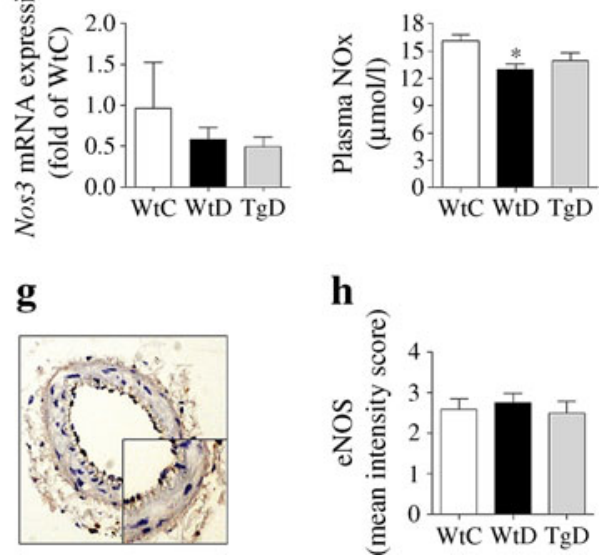

metabolites nitrite and nitrate (NOx) were significantly decreased by diabetes but not in Glol-overexpressing rats (d). (e-h) Diabetes and Glol overexpression did not result in altered eNOS protein levels as shown by immunohistochemical staining (e, WtC; $\mathbf{f}, \mathrm{WtD} ; \mathbf{g}, \mathrm{TgD}$ rats) and its quantification (h). Scale bars in (e) $100 \mu \mathrm{m}$ in the main image and $50 \mu \mathrm{m}$ in the insert

the diabetes-induced increase in urinary nephrin $(p=0.22)$, another marker for podocyte loss (Fig. 5i).

In line with the in vivo data, we observed that in fully differentiated cultured podocytes incubation with $1,000 \mu \mathrm{mol} / 1 \mathrm{MGO}$ for $48 \mathrm{~h}$ resulted in an increase in apoptotic cells compared with control cells (Fig. 5j). Early and late apoptotic cells were quantified with annexin-V and propidium iodide FACS, respectively (Fig. 5k). These data indicate that the apoptosis-induced decrease in podocytes in the diabetic glomerulus can at least be partially caused by MGO cytotoxicity.
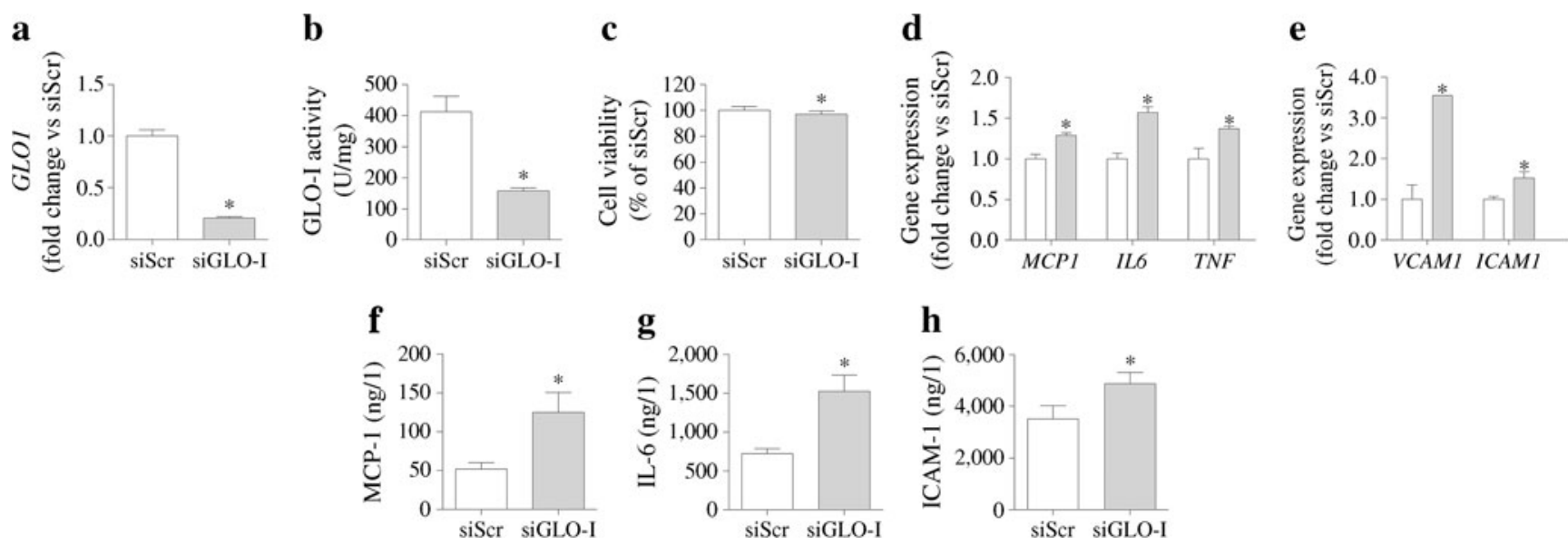

Fig. 4 Transfection of cultured human endothelial cells with siRNA against GLO1 (siGLO-I) resulted in a significant $\left({ }^{*} p<0.05\right.$, compared with scrambled siRNA [siScr]) decrease in GLO1 expression (a) and activity (b). This resulted in an almost $10 \%$ decrease in cell viability $\left({ }^{*} p<0.05\right.$, compared with siScrambled), as measured by MTT assay (c).

Furthermore several genes involved in inflammation $(M C P 1$ [also known as CCL2 ], IL6 and $T N F \alpha)(\mathbf{d})$ and endothelial activation (VCAMI and ICAM1) (e) were upregulated by siGLO-I. Upregulation of gene expression was confirmed by increased protein levels of MCP-1 (f), IL-6 (g) and ICAM-1 (h) in the cell supernatant fractions 
Table 2 Renal characteristics of the rats after 24 weeks of diabetes

\begin{tabular}{llll}
\hline Renal characteristic & WtC & WtD & TgD \\
\hline Left kidney weight $(\mathrm{g})$ & $1.4 \pm 0.1$ & $2.0 \pm 0.1^{*}$ & $1.8 \pm 0.1^{*}$ \\
Right kidney weight $(\mathrm{g})$ & $1.5 \pm 0.1$ & $2.0 \pm 0.1^{*}$ & $1.8 \pm 0.1^{*}$ \\
Left kidney/body weight ratio (mg/g) & $3.0 \pm 0.1$ & $6.6 \pm 0.2^{*}$ & $6.1 \pm 0.1^{*}$ \\
Right kidney/body weight ratio (mg/g) & $3.1 \pm 0.1$ & $6.5 \pm 0.2^{*}$ & $6.1 \pm 0.2^{*}$ \\
$\begin{array}{l}\text { Glomerular volume }\left(\mathrm{mm}^{3}\right) \\
\text { Creatinine clearance } \\
\quad\left(\mathrm{ml} \mathrm{h}^{-1}[100 \mathrm{~g}]^{-1}\right)\end{array}$ & $86 \pm 8$ & $129 \pm 14^{*}$ & $103 \pm 16$ \\
& & $103 \pm 40$ & $71 \pm 6$ \\
\end{tabular}

$* p<0.05$ compared with $\mathrm{WtC}$

To address the effects of these diabetes-induced glomerular alterations on the filtration barrier function we next determined the presence of early markers of renal damage in the urine of the rats. Urinary albumin, lipocalin-2, osteopontin and KIM-1 were all elevated after 24 weeks of diabetes (Fig. 6a-d). This elevation was attenuated for three of the four early markers (albumin, osteopontin and KIM-1) in rats overexpressing Glo1 . The composite score for albumin, lipocalin-2, osteopontin and KIM-1 was significantly increased in the wild-type diabetic group and Glo overexpression decreased this combination of renal injury markers (Fig. 6e).

\section{Discussion}

In this study we showed that diabetes-induced impairment of vascular function, as measured by an impaired endothelium-
Fig. 5 Podocyte number and number of apoptotic cells were determined by WT-1 and activecaspase-3 staining, respectively. Compared with control rats (a), wild-type diabetic rats (b) showed significantly $(* * p<0.01)$ fewer numbers of podocytes per glomerulus. Overexpression of Glo1 could attenuate this $\left({ }^{\dagger} p<0.05\right.$ compared with wild-type diabetic, WtD) (c), as shown by the quantification (d). The number of apoptotic cells was significantly $(* p<0.05)$ increased after 24 weeks of diabetes (f) compared with controls (e). This increase was prevented by Glo 1 overexpression $\left({ }^{\dagger} p<0.05\right.$, compared with WtD) $(\mathbf{g}, \mathbf{h})$. Scale bar in (a) $100 \mu \mathrm{m}$. In line with this, the urinary podocyte marker nephrin was mildly elevated in the urine of the wildtype diabetic rats (i). In cultured podocytes, incubation with 1 , $000 \mu \mathrm{mol} / \mathrm{l} \mathrm{MGO}$ for $48 \mathrm{~h}$ resulted in an increase of both annexin-V (AnnV)-positive and AnnV/propidium iodide (PI)double-positive cells compared with control cells. (j)

Representive FACS data of control (-MGO) and MGOincubated cells

(+MGO). (k) Quantification of the AnnV-positive cells (white bars), AnnV/PI-double-positive cells (grey bars) and the combined AnnV-positive population (black bars) $(* * * p<0.001$ compared with control) $\mathbf{a}$

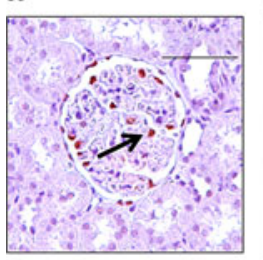

b

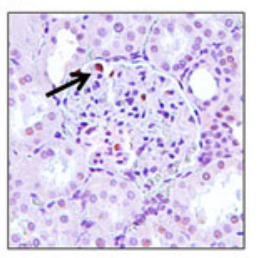

c

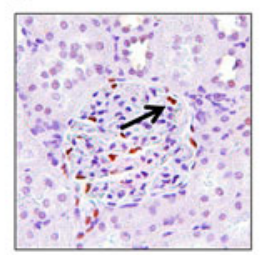

f

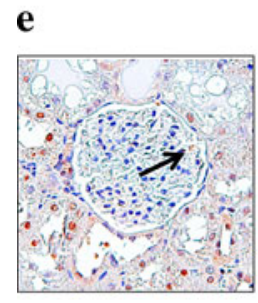

i

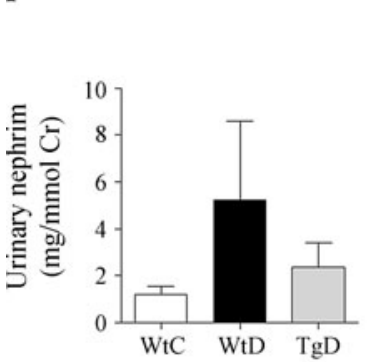

$\mathbf{k}$
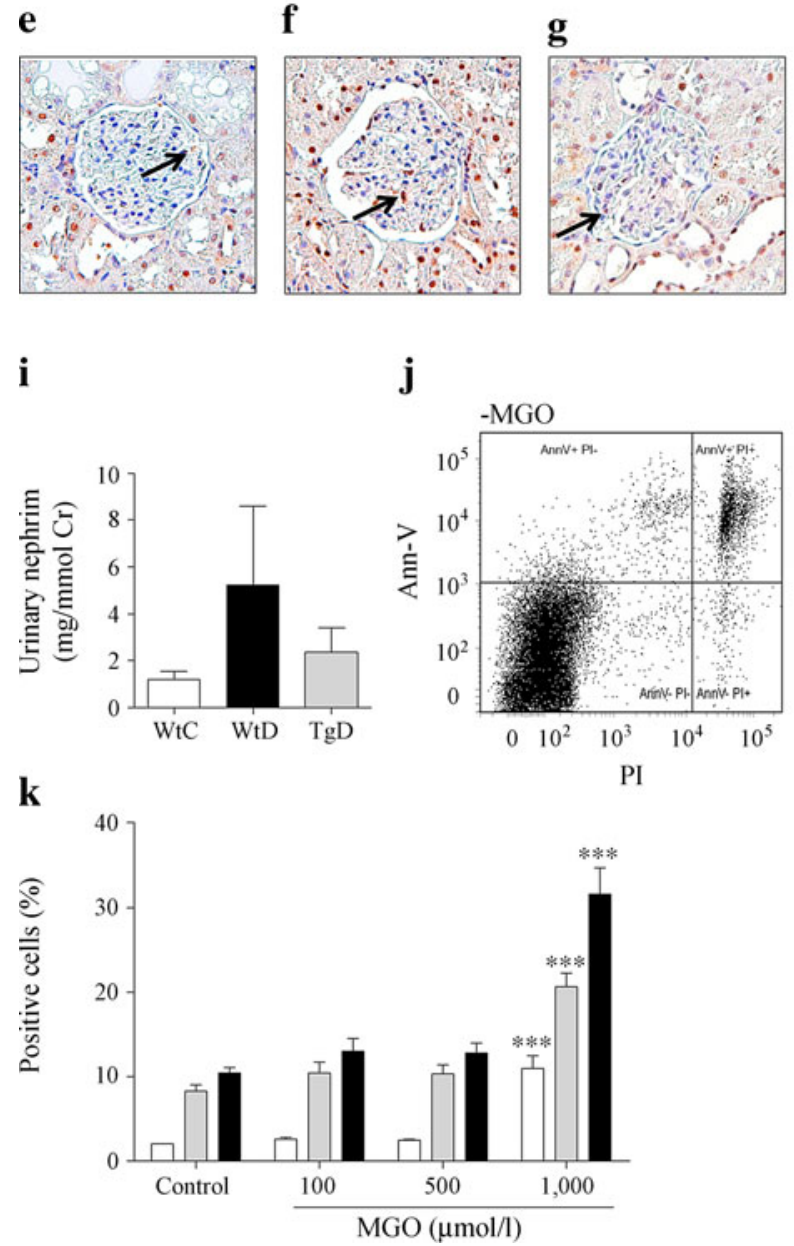

d

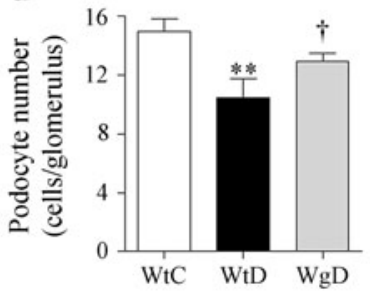

h

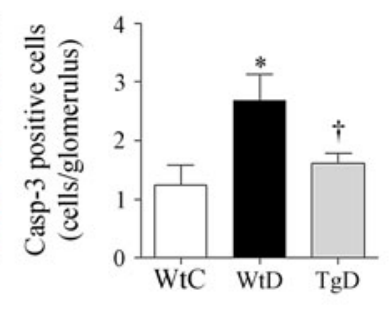

j
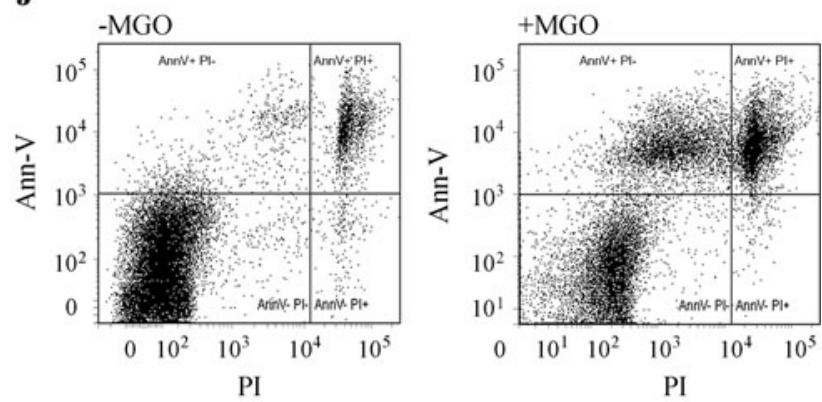

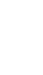



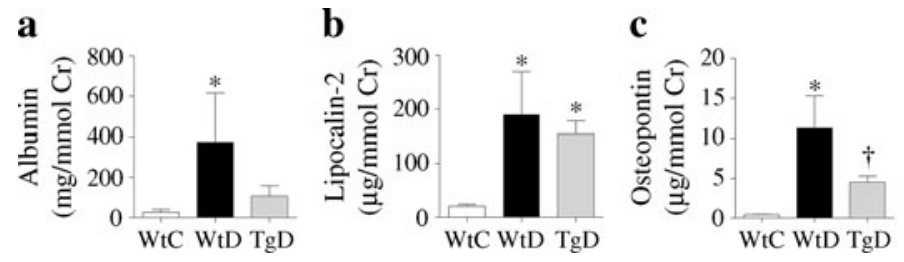
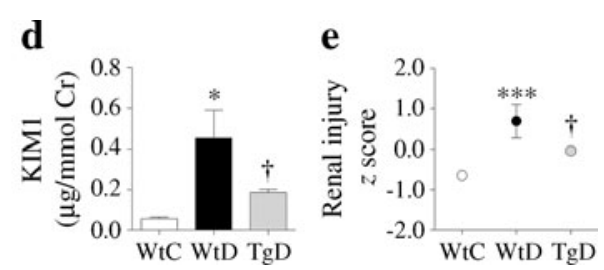

Fig. 6 Twenty-four weeks of diabetes increased the levels of albumin (a), lipocalin-2 (b), osteopontin (c) and KIM-1 (d) (all *p $p 0.05$ compared with wild-type control, WtC), and this elevation was significantly attenuated by Glo1 overexpression for osteopontin and KIM-1 ( ${ }^{\dagger} p<0.05$ compared with wild-type diabetes, WtD). Lipocalin-2 was still significantly elevated in the TgD animals compared with $\mathrm{WtD}$ $\left({ }^{*} p<0.05\right)$, but the statistical significant diabetes-induced increase in albumin was lost due to Glo1 overexpression. After 24 weeks of diabetes the renal injury composite score (albumin, lipocalin-2, osteopontin and KIM-1) was significantly increased $(* * * p<0.001$ compared with $\mathrm{WtC}$ ), and could be partially decreased by Glol overexpression $\left({ }^{\dagger} p<0.05\right.$ compared with WtD) dependent relaxation and an increased expression of markers for endothelial dysfunction, in mesenteric arteries of diabetic rats is prevented by overexpression of Glo1. In line with this, downregulation of Glol in cultured human endothelial cells resulted in a pro-inflammatory and endothelial-activated profile. In addition, overexpression of Glol attenuated loss of podocytes in the glomerulus and also renal excretion of early markers of diabetic nephropathy. MGO-induced podocyte apoptosis and generation of AGEs in the vasculature seem to contribute to this pathogenesis. These data demonstrate a direct link between increased levels of AGEs, endothelial dysfunction and the development of renal changes in experimental diabetes.

Our results showed that the diabetes-induced elevation of the AGEs CML and MG-H1 in vascular tissue is prevented by overexpression of Glo1. This decrease in vascular tissue levels of MGO and GO-derived AGEs demonstrates the efficiency of Glol overexpression in the detoxification of both GO and MGO. This is in accordance with our previous study in which we demonstrated that Glol overexpression prevents elevated blood levels of GO and MGO, and thereby decreases plasma AGE levels after 12 weeks of diabetes [13]. In contrast, the CML levels in the plasma could not be significantly decreased by Glo1 overexpression after 24 weeks of diabetes, suggesting that an excessive formation of CML via additional pathways, like lipid peroxidation, possibly exceeds the detoxification rate in this prolonged state of diabetes.

We previously addressed the reaction of the mesenteric endothelium to acetylcholine after short-time incubations with MGO or high glucose and in diabetic rats 12 weeks after STZ injection [14]. In the current study we confirmed these data in mesenteric arteries after 24 weeks of diabetes and extended our observations by specifically addressing which pathways are involved in the diabetes-induced impairment of acetylcholine-dependent relaxation. Our approach revealed that the decreased endothelium-dependent relaxation is caused by impairment of both the eNOS/NO pathway and endothelium-derived hyperpolarising factors. In contrast with several studies suggesting a decrease in expression and activity of the eNOS enzyme [22, 23], our results show that its mRNA and protein levels were not altered by diabetes. Unfortunately, we did not address the phosphorylation state of eNOS, therefore we could not attribute the impairment of eNOS activity to decreased phosphorylation, as Dhar et al described [24]. However, we previously showed that MGO and MGO-arginine adducts do not directly inhibit the overall eNOS activity in vitro [25]. Furthermore the plasma levels of the NO metabolites nitrite and nitrate were significantly decreased in the wild-type diabetic rats, indicating a decrease in NO bioavailability rather than reduced NO production. Interestingly, mRNA levels of the genes encoding oxidative stress-sensing master switch nuclear factor (erythroid-derived 2)-like 2 (NRF-2), and NRF-2-responsive detoxifying genes Glo1, NADPH-oxidase-4 and sodium oxide dismutase-3, were increased in the wild-type diabetic rats (data not shown), and this oxidative stress response was absent in the GLO-I diabetic rats, suggesting that diabetes-induced quenching of NO by oxidative stress occurred.

Elevated levels of cellular adhesion molecules are associated with an increase in leucocyte adhesion and inflammatory activation [26]. Our results showed that Glo1 overexpression prevented the STZ-induced increase of both Vcam1 and Icam1 expression. In line with this observation, our experiments in cultured endothelial cells showed that GLO-I is an important regulator in the prevention of endothelial inflammation and activation. These results confirmed the link between markers of endothelial function and AGEs [27, 28].

Diabetes is characterised by hypertrophy of several target tissues including the kidneys and the vasculature [29, 30]. Consistent with these findings, we detected an increase in kidney weight and glomerular size after 24 weeks of diabetes. Overexpression of Glol revealed a trend of decreasing the renal hypertrophy, although not statistically significant. We did not see any differences in morphological lesions of the glomeruli even after 24 weeks of diabetes, which is in accordance with earlier studies in STZ rats. Hirose et al [31] reported that basal membrane thickness is the only variable of advanced diabetic glomerulopathy that increases significantly 
in STZ rats, albeit only after more than 6 months of diabetes. Bidani et al [32] speculated that blood pressure reduction in STZ rats is one of the reasons that the nephrotic damage observed in the STZ diabetes model is only modest. Indeed, in our model we also observed a decrease in blood pressure in the diabetic rats and, interestingly, Glol overexpression significantly blunted the diabetes-induced blood pressure depression.

In humans, there is convincing evidence that endothelial dysfunction is closely associated with the development of diabetic nephropathy [33, 34]. In type 1 diabetes, microalbuminuria and macroalbuminuria are accompanied by a variety of markers of endothelial dysfunction, such as increased plasma concentrations of VCAM-1, endothelin-1 and plasminogen activator inhibitor-1, and an impaired endothelium-dependent, NO-mediated vasorelaxation [35]. Consistent with this, we observed an increase in albuminuria in diabetes, which showed a tendency to be improved by Glo1 overexpression. In addition to albumin (which was not statistically significant), two other markers for nephropathy, KIM-1 and osteopontin, were significantly improved by Glo1 overexpression. Although the function of KIM-1 is unclear, its abundant tubular expression after damage points to a role in either tubular damage or repair [36]. Also osteopontin, a large phosphoglycoprotein adhesion molecule, is upregulated in diabetic nephropathy. A recent study by Nicholas et al suggests that osteopontin is a key profibrotic factor that contributes to the development of glomerulosclerosis and albuminuria in diabetes [37].

Diabetic nephrotic injuries of both the glomeruli and the tubulointerstitium are accompanied by increased protein excretion and consequently by a decline in renal function [38]. It is likely that diabetic nephropathy occurs as a result of an interplay between haemodynamic and metabolic factors [39]. Thus, endothelial dysfunction, as an early phenomenon, may explain the typical association between albuminuria and extra-renal complications. It may be speculated that endothelial dysfunction, as we observed in the rat mesenteric arteries, is also present in the intra-renal vasculature [40, 41], thereby resulting in increased pressure in the glomeruli, which consequently leads to glomerular dysfunction and urinary protein leakage. Our observations that Glo1 overexpression reduced diabetes-induced vascular and renal dysfunction, as well as data from other studies [30, 42, 43], support a role for glycation in these pathogeneses. In addition, a cohort study by Beisswenger et al showed that progression of diabetic nephropathy is also significantly related to elevation in dicarbonyl stress [11]. In this study elevated levels of MGO correlated with loss of podocytes, another mechanism preceding glomerular leakage [44]. This strengthens our observations that MGO induces podocyte apoptosis and that diabetes-induced podocyte loss can be prevented by Glol overexpression.
In summary, this study showed for the first time that overexpression of Glo1 reduces diabetes-induced endothelial dysfunction and attenuates early renal impairment in an animal model of diabetes. These observations suggest that inhibition of intracellular glycation prevents deterioration of vascular and renal function in experimental diabetes. A recent study with the angiotensin type 1 receptor blocker candesartan showed that GLO-I activity can be positively regulated by drug intervention [45]. Therefore activation of GLO-I may provide an approach to prevent endothelial dysfunction and vascular complications in diabetes.

Acknowledgements The authors gratefully acknowledge M. van den Waarenburg and J. Scheijen (Department of Internal Medicine, Division of General Internal Medicine, Laboratory for Metabolism and Vascular Medicine, Maastricht University, Maastricht, the Netherlands) for the measurement of the urinary kidney injury markers, soluble adhesion molecules and plasma/tissue AGE levels. Furthermore we also acknowledge J. Debets (Department of Pharmacology, Maastricht University, Maastricht, the Netherlands) for the measurement of intraarterial blood pressure in the rats.

Funding This study was partially supported by the Dutch Diabetes Foundation (Grant number 2005.11.013) and further supported by the Swiss National Science Foundation Fellowship PBBSP3-144160 (JS), National Institutes Health Grants DK62472 and DK57683 (PM) and the Danish Diabetes Academy supported by the Novo Nordisk Foundation (JAØ).

Duality of interest The authors declare that there is no duality of interest associated with this manuscript.

Contribution statement $\mathrm{OB}, \mathrm{AF}, \mathrm{CJPK}, \mathrm{BJAJ}, \mathrm{JGRDM}$ and CGS designed the study and experiments. TM, MB, PHM and CDAS contributed experimental and conceptual expertise. OB, PMGN, JAØ, and JS performed the research. All authors analysed, interpreted and discussed the data. OB and CGS wrote the manuscript. All authors reviewed and revised the manuscript critically. All authors approved the final version of the manuscript.

\section{References}

1. Skyler JS, Bergenstal R, Bonow RO et al (2009) Intensive glycemic control and the prevention of cardiovascular events: implications of the ACCORD, ADVANCE, and VA diabetes trials: a position statement of the American Diabetes Association and a scientific statement of the American College of Cardiology Foundation and the American Heart Association. Circulation 119:351-357

2. Clark CM Jr, Lee DA (1995) Prevention and treatment of the complications of diabetes mellitus. N Engl J Med 332:1210-1217

3. Klein R (1995) Hyperglycemia and microvascular and macrovascular disease in diabetes. Diabetes Care 18:258-268

4. Cines DB, Pollak ES, Buck CA et al (1998) Endothelial cells in physiology and in the pathophysiology of vascular disorders. Blood 91:3527-3561

5. Brownlee M (2001) Biochemistry and molecular cell biology of diabetic complications. Nature 414:813-820

6. Monnier VM, Cerami A (1981) Nonenzymatic browning in vivo: possible process for aging of long-lived proteins. Science 211:491493 
7. Brownlee M (2005) The pathobiology of diabetic complications: a unifying mechanism. Diabetes 54:1615-1625

8. Shinohara M, Thornalley PJ, Giardino I et al (1998) Overexpression of glyoxalase-I in bovine endothelial cells inhibits intracellular advanced glycation endproduct formation and prevents hyperglycemia-induced increases in macromolecular endocytosis. J Clin Invest 101:1142-1147

9. Thornalley PJ (1993) The glyoxalase system in health and disease. Mol Aspects Med 14:287-371

10. Rosca MG, Mustata TG, Kinter MT et al (2005) Glycation of mitochondrial proteins from diabetic rat kidney is associated with excess superoxide formation. Am J Physiol Renal Physiol 289:F420F430

11. Beisswenger PJ, Drummond KS, Nelson RG, Howell SK, Szwergold BS, Mauer M (2005) Susceptibility to diabetic nephropathy is related to dicarbonyl and oxidative stress. Diabetes 54:3274-3281

12. Ogawa S, Nakayama K, Nakayama M et al (2010) Methylglyoxal is a predictor in type 2 diabetic patients of intima-media thickening and elevation of blood pressure. Hypertension 56:471-476

13. Brouwers O, Niessen PM, Ferreira I et al (2011) Overexpression of glyoxalase-I reduces hyperglycemia-induced levels of advanced glycation endproducts and oxidative stress in diabetic rats. J Biol Chem 286:7

14. Brouwers O, Niessen PM, Haenen G et al (2010) Hyperglycaemiainduced impairment of endothelium-dependent vasorelaxation in rat mesenteric arteries is mediated by intracellular methylglyoxal levels in a pathway dependent on oxidative stress. Diabetologia 53:9891000

15. Berner AK, Brouwers O, Pringle R et al (2012) Protection against methylglyoxal-derived AGEs by regulation of glyoxalase 1 prevents retinal neuroglial and vasodegenerative pathology. Diabetologia 55: $845-854$

16. McLellan AC, Phillips SA, Thornalley PJ (1993) The assay of S-Dlactoylglutathione in biological systems. Biochem Soc Trans 21: $164 \mathrm{~S}$

17. Schalkwijk CG, Baidoshvili A, Stehouwer CD, van Hinsbergh VW, Niessen HW (2004) Increased accumulation of the glycoxidation product Nepsilon-(carboxymethyl)lysine in hearts of diabetic patients: generation and characterisation of a monoclonal anti-CML antibody. Biochim Biophys Acta 1636:82-89

18. Ostergaard JA, Bjerre M, RamachandraRao SP et al (2012) Mannan-binding lectin in diabetic kidney disease: the impact of mouse genetics in a type 1 diabetes model. Exp Diabetes Res 2012:678381

19. Waterval WA, Scheijen JL, Ortmans-Ploemen MM, Habets-van der Poel CD, Bierau J (2009) Quantitative UPLC-MS/MS analysis of underivatised amino acids in body fluids is a reliable tool for the diagnosis and follow-up of patients with inborn errors of metabolism. Clin Chim Acta 407:36-42

20. Hanssen NM, Engelen L, Ferreira I et al (2013) Plasma levels of advanced glycation endproducts Nepsilon-(carboxymethyl) lysine, Nepsilon-(carboxyethyl)lysine, and pentosidine are not independently associated with cardiovascular disease in individuals with or without type 2 diabetes: the Hoorn and CODAM studies. J Clin Endocrinol Metab 98:E1369-1373

21. Scheijen JL, Schalkwijk CG (2013) Quantification of glyoxal, methylglyoxal and 3-deoxyglucosone in blood and plasma by ultra performance liquid chromatography tandem mass spectrometry: evaluation of blood specimen. Clin Chem Lab Med 13:1-7

22. Nagareddy PR, Xia Z, MacLeod KM, McNeill JH (2006) NAcetylcysteine prevents nitrosative stress-associated depression of blood pressure and heart rate in streptozotocin diabetic rats. J Cardiovasc Pharmacol 47:513-520

23. Hink U, Li H, Mollnau H et al (2001) Mechanisms underlying endothelial dysfunction in diabetes mellitus. Circ Res 88:E14-E22
24. Dhar A, Dhar I, Desai KM, Wu L (2010) Methylglyoxal scavengers attenuate endothelial dysfunction induced by methylglyoxal and high concentrations of glucose. Br J Pharmacol 161:1843-1856

25. Brouwers O, Teerlink T, van Bezu J, Barto R, Stehouwer CD, Schalkwijk CG (2008) Methylglyoxal and methylglyoxal-arginine adducts do not directly inhibit endothelial nitric oxide synthase. Ann N Y Acad Sci 1126:231-234

26. Tailor A, Granger DN (2000) Role of adhesion molecules in vascular regulation and damage. Curr Hypertens Rep 2:78-83

27. Nin JW, Jorsal A, Ferreira I et al (2011) Higher plasma levels of advanced glycation end products are associated with incident cardiovascular disease and all-cause mortality in type 1 diabetes: a 12-year follow-up study. Diabetes Care 34:442447

28. Vlassara H, Fuh H, Donnelly T, Cybulsky M (1995) Advanced glycation endproducts promote adhesion molecule (VCAM-1, ICAM-1) expression and atheroma formation in normal rabbits. Mol Med 1:447-456

29. Chang KC, Hsu KL, Tseng CD, Lin YD, Cho YL, Tseng YZ (2006) Aminoguanidine prevents arterial stiffening and cardiac hypertrophy in streptozotocin-induced diabetes in rats. Br J Pharmacol 147:944950

30. Soulis T, Sastra S, Thallas V et al (1999) A novel inhibitor of advanced glycation end-product formation inhibits mesenteric vascular hypertrophy in experimental diabetes. Diabetologia 42: $472-479$

31. Hirose K, Osterby R, Nozawa M, Gundersen HJ (1982) Development of glomerular lesions in experimental long-term diabetes in the rat. Kidney Int 21:689-695

32. Bidani AK, Picken M, Hacioglu R, Williamson G, Griffin KA (2007) Spontaneously reduced blood pressure load in the rat streptozotocininduced diabetes model: potential pathogenetic relevance. Am J Physiol Renal Physiol 292:F647-F654

33. Astrup AS, Tarnow L, Pietraszek L et al (2008) Markers of endothelial dysfunction and inflammation in type 1 diabetic patients with or without diabetic nephropathy followed for 10 years: association with mortality and decline of glomerular filtration rate. Diabetes Care 31:1170-1176

34. Stehouwer CD, Stroes ES, Hackeng WH, Mulder PG, Den Ottolander GJ (1991) von Willebrand factor and development of diabetic nephropathy in IDDM. Diabetes 40:971-976

35. Schalkwijk CG, Stehouwer CD (2005) Vascular complications in diabetes mellitus: the role of endothelial dysfunction. Clin Sci (Lond) 109:143-159

36. Ichimura T, Bonventre JV, Bailly V et al (1998) Kidney injury molecule-1 (KIM-1), a putative epithelial cell adhesion molecule containing a novel immunoglobulin domain, is upregulated in renal cells after injury. J Biol Chem 273:41354142

37. Nicholas SB, Liu J, Kim J et al (2010) Critical role for osteopontin in diabetic nephropathy. Kidney Int 77:588-600

38. Mauer SM, Steffes MW, Ellis EN, Sutherland DE, Brown DM, Goetz FC (1984) Structural-functional relationships in diabetic nephropathy. J Clin Invest 74:1143-1155

39. Cooper ME (2001) Interaction of metabolic and haemodynamic factors in mediating experimental diabetic nephropathy. Diabetologia 44:1957-1972

40. Dai FX, Diederich A, Skopec J, Diederich D (1993) Diabetesinduced endothelial dysfunction in streptozotocin-treated rats: role of prostaglandin endoperoxides and free radicals. J Am Soc Nephrol 4:1327-1336

41. De Vriese AS, Van de Voorde J, Blom HJ, Vanhoutte PM, Verbeke M, Lameire NH (2000) The impaired renal vasodilator response attributed to endothelium-derived hyperpolarizing factor in streptozotocin-induced diabetic rats is restored by 5methyltetrahydrofolate. Diabetologia 43:1116-1125 
42. Forbes JM, Thallas V, Thomas MC et al (2003) The breakdown of preexisting advanced glycation end products is associated with reduced renal fibrosis in experimental diabetes. FASEB J 17:17621764

43. Soulis T, Cooper ME, Vranes D, Bucala R, Jerums G (1996) Effects of aminoguanidine in preventing experimental diabetic nephropathy are related to the duration of treatment. Kidney Int 50:627-634
44. Siu B, Saha J, Smoyer WE, Sullivan KA, Brosius FC 3rd (2006) Reduction in podocyte density as a pathologic feature in early diabetic nephropathy in rodents: prevention by lipoic acid treatment. BMC Nephrol 7:6

45. Miller AG, Tan G, Binger KJ et al (2010) Candesartan attenuates diabetic retinal vascular pathology by restoring glyoxalase-I function. Diabetes 59:3208-3215 